\title{
Constrained Lipschitzian Error Bounds and Noncritical Solutions of Constrained Equations
}

\author{
A. Fischer ${ }^{1}$ (D) . A. F. Izmailov ${ }^{2}$ (D) M. Jelitte ${ }^{1}$ (D)
}

Received: 9 March 2020 / Accepted: 20 October 2020 / Published online: 9 November 2020

(C) The Author(s) 2020

\begin{abstract}
For many years, local Lipschitzian error bounds for systems of equations have been successfully used for the design and analysis of Newton-type methods. There are characterizations of those error bounds by means of first-order derivatives like a recent result by Izmailov, Kurennoy, and Solodov on critical solutions of nonlinear equations. We aim at extending this result in two directions which shall enable, to some extent, to include additional constraints and to consider mappings with reduced smoothness requirements. This leads to new necessary as well as sufficient conditions for the existence of error bounds.
\end{abstract}

Keywords Constrained equation · constrained error bound $\cdot$ critical and noncritical solutions $\cdot$ (piecewise) semidifferentiable functions

Mathematics Subject Classification (2010) 49J52 · 49J53 · 90C33

The work was funded by the Volkswagen Foundation, by the Deutsche Forschungsgemeinschaft (DFG, German Research Foundation) - 409756759, and by the Russian Foundation for Basic Research Grants 19-51-12003 NNIO_a and 20-01-00106.

Dedicated to Professor R. Tyrrell Rockafellar on the occasion of his 85th birthday.

M. Jelitte

Mario.Jelitte@tu-dresden.de

A. Fischer

Andreas.Fischer@tu-dresden.de

A. F. Izmailov

izmaf@ccas.ru

1 Faculty of Mathematics, Technische Universität Dresden, 01062 Dresden, Germany

2 VMK Faculty, OR Department, Lomonosov Moscow State University (MSU), Uchebniy Korpus 2, Leninskiye Gory, 119991 Moscow, Russia 


\section{Introduction}

The present article focuses on the characterization of constrained local Lipschitzian error bounds. More in detail, let a set $\Omega \subset \mathbb{R}^{n}$ and a function $f: \mathbb{R}^{n} \rightarrow[0, \infty]$ be given such that

$$
U:=f^{-1}(0) \cap \Omega
$$

is the nonempty solution set of the constrained system

$$
f(u)=0 \quad \text { s.t. } \quad u \in \Omega .
$$

We say that $f$ provides a local $\Omega$-error bound at $u^{*} \in U$ for $U$, if there are constants $c, \varepsilon>0$ such that

$$
c \text { dist }[u, U] \leq f(u) \text { for all } u \in \Omega \cap\left(u^{*}+\varepsilon \mathbb{B}\right),
$$

where the distance of $u \in \mathbb{R}^{n}$ to a nonempty set $W \subset \mathbb{R}^{n}$ is given by

$$
\operatorname{dist}[u, W]:=\inf \{\|u-w\| \mid w \in W\},
$$

while $\|\cdot\|$ stands for the Euclidean norm, and $\mathbb{B}:=\left\{u \in \mathbb{R}^{n} \mid\|u\| \leq 1\right\}$ denotes the unit ball.

Obviously, the inequality in (3) is restricted to some local neighborhood of $u^{*}$ intersected with the constraint set $\Omega$, so that this property is also called constrained local Lipschitzian error bound. For brevity, we will omit the term "Lipschitzian" throughout. In the special case when $\Omega=\mathbb{R}^{n}$, the property in question will be simply called local error bound.

The function $f$ may come from different applications. For example,

$$
f(u):=\|F(u)\|
$$

is often considered for some given mapping $F: \mathbb{R}^{n} \rightarrow \mathbb{R}^{m}$. Another application can be found in constrained minimization problems

$$
f_{0}(u) \rightarrow \min \quad \text { s.t. } \quad u \in \Omega
$$

by setting $f(u):=f_{0}(u)-f_{*}$, where $f_{*} \in \mathbb{R}$ denotes the minimal value of $f$ on $\Omega$ (if it exists), see [7].

Global and local error bounds are known to play a fundamental role in mathematical programming and, particularly, in the design and analysis of numerical methods. For more details, the reader is referred to a survey [28] published in 1997. Since that time growing attention has been directed on local error bounds. In particular, it turned out that local error bounds may help to establish a superlinear rate of convergence for Newton-type methods in the absence of nonsingularity assumptions. For early works see [10, 17, 36] in the context of constrained optimization with nonisolated multipliers, and [11, 37] for (generalized) equations with nonisolated solutions. In the meanwhile, several advances have been made. For example, this includes the use of constrained local error bounds [2, 3, 23] in the sense of (3). It was shown in $[4,8,9]$ that constrained local error bounds are particularly useful if a loss of nonsingularity comes together with nonsmoothness. Last but not least, we would like to mention articles, where conditions for the existence of a local error bound were derived for special problem classes [13, 14, 16].

An approach to characterize the existence of a local error bound for an unconstrained system

$$
F(u)=0
$$

of differentiable equations was given in [20]. There, in generalization of the concept of a noncritical Lagrange multiplier (see [19, 22], and [26] for recent advances), and answering the question raised in [12], the notion of a noncritical solution $u^{*}$ of (5) is introduced. 
Under a certain strict differentiability assumption, the equivalence of the noncriticality of a solution $u^{*}$ and the property that $f$ defined in (4) provides a local error bound at $u^{*}$ for $F^{-1}(0)$ is shown. However, it turned out to be challenging [1] to characterize a constrained error bound by (a reasonable notion of) a noncritical solution of a constrained system of equations.

The present paper is devoted to the latter problem in a nonsmooth setting. In Section 2, we provide some necessary preliminaries, like a discussion of the notions of semidifferentiability to be used in the paper, and related approximations of the (regular) tangent cone to the solution set. Our main results are presented in Section 3. In particular, Section 3.1 contains necessary conditions for a local $\Omega$-error bound, while Section 3.2 contains the corresponding sufficient conditions, both not relying on any differentiability assumptions (even generalized). These conditions can be regarded as an extension of the characterization of a local error bound as a noncritical solution, established in [20]. In Section 3.3, employing semidifferentiability, we propose a characterization of a local $\Omega$-error bound in a way that is inspired by Mordukhovich's criterion for the Aubin property. This gives an alternative understanding of noncriticality. We will discuss our main results for some special cases in Section 4. An application to constrained piecewise strictly semidifferentiable systems of equations is finally given in Section 5 .

Some words about our notation and blanket assumptions. Throughout the paper, the set $U$ is defined as in (1), $u^{*} \in U$ is arbitrary but fixed, and $U$ is assumed closed near $u^{*}$. (The latter holds automatically if $\Omega$ is closed and $f$ is lower semicontinuous on $\Omega$ near $u^{*}$.) Let $\mathbb{S}:=\left\{u \in \mathbb{R}^{n} \mid\|u\|=1\right\}$ denote the unit sphere. A nonempty set $C \subset \mathbb{R}^{n}$ is called cone if $u \in C$ implies $\lambda u \in C$ for all $\lambda \geq 0$. By $C^{\circ}:=\left\{v \in \mathbb{R}^{n} \mid v^{\top} u \leq 0 \forall u \in C\right\}$, the polar of a cone $C$ is denoted. For a set $W \subset \mathbb{R}^{n}$ and $u \in W$, we write $u^{\prime} \stackrel{W}{\rightarrow} u$ to say that any sequence $\left(u^{k}\right) \subset W$ with $u^{k} \rightarrow u$ is meant. Moreover, $o:(0, \infty) \rightarrow \mathbb{R}$ denotes a function with the property $o(t) / t \rightarrow 0$ as $t \downarrow 0$. The null space of a linear mapping $A: \mathbb{R}^{n} \rightarrow \mathbb{R}^{m}$ is defined as $\operatorname{ker} A:=\left\{v \in \mathbb{R}^{n} \mid A v=0\right\}$.

\section{Preliminaries}

In [20, formula (8)], a slightly more special version of the following notion was introduced.

Definition 1 (Strict differentiability with respect to a set) Let a set $W \subset \mathbb{R}^{n}$ and $\widehat{u} \in W$ be given. A mapping $F: \mathbb{R}^{n} \rightarrow \mathbb{R}^{m}$ is called strictly differentiable at $\widehat{u}$ with respect to $W$ if $F$ is differentiable at $\widehat{u}$ and

$$
\left\|F(u+v)-F(u)-F^{\prime}(\widehat{u}) v\right\|=o(\|v\|) \quad \text { as } u \stackrel{W}{\rightarrow} \widehat{u} \text { and } v \rightarrow 0 .
$$

Motivated by this definition, and by the notion of semidifferentiable functions in [33, Definition 7.20], we introduce the following two notions of directional differentiability, both weaker than the one in Definition 1.

Definition 2 (Semidifferentiability) Let a cone $C \subset \mathbb{R}^{n}$ and $\widehat{u} \in \mathbb{R}^{n}$ be given. A mapping $F: \mathbb{R}^{n} \rightarrow \mathbb{R}^{m}$ is called semidifferentiable at $\widehat{u}$ for $C$ if, for each $v \in C$, there exists some $F^{\prime}(\widehat{u} ; v) \in \mathbb{R}^{m}$ such that

$$
\left\|F\left(\widehat{u}+t v^{\prime}\right)-F(\widehat{u})-t F^{\prime}(\widehat{u} ; v)\right\|=o(t) \quad \text { as } t \downarrow 0 \text { and } v^{\prime} \stackrel{C}{\rightarrow} v .
$$

If the latter holds true for $C=\mathbb{R}^{n}$, we simply call $F$ semidifferentiable at $\widehat{u}$. 
Definition 3 (Strict semidifferentiability with respect to a set) Let a cone $C \subset \mathbb{R}^{n}$, a set $W \subset \mathbb{R}^{n}$, and $\widehat{u} \in W$ be given. A mapping $F: \mathbb{R}^{n} \rightarrow \mathbb{R}^{m}$ is called strictly semidifferentiable at $\widehat{u}$ with respect to $W$ for $C$ if, for each $v \in C$, there exists some $F^{\prime}(\widehat{u} ; v) \in \mathbb{R}^{m}$ such that

$$
\left\|F\left(u+t v^{\prime}\right)-F(u)-t F^{\prime}(\widehat{u} ; v)\right\|=o(t) \quad \text { as } u \stackrel{W}{\rightarrow} \widehat{u}, t \downarrow 0, \text { and } v^{\prime} \rightarrow v .
$$

If the latter holds true for $C=\mathbb{R}^{n}$, we say that $F$ is strictly semidifferentiable at $\widehat{u}$ with respect to $W$.

Note that for any $v \in C$, the vector $F^{\prime}(\widehat{u} ; v)$ in these definitions necessarily coincides with the standard directional derivative of $F$ at $\widehat{u}$ in the direction $v$, i.e.,

$$
F^{\prime}(\widehat{u} ; v)=\lim _{t \downarrow 0} \frac{F(\widehat{u}+t v)-F(\widehat{u})}{t} .
$$

In particular, $F^{\prime}(\widehat{u} ; v)$ is thus uniquely defined. If $F$ is differentiable at $\widehat{u}$ and $C=\mathbb{R}^{n}$, then $F^{\prime}(\widehat{u})$ coincides with the linear mapping associated with the Jacobian. Observe also that the larger $C$ or $W$ are, the more restrictive Definitions 2 and 3 become. Moreover, the property specified in Definition 1 implies the one in Definition 3 (with any $C$ ), while the property specified in Definition 3 implies the one in Definition 2, with the same $F^{\prime}(\widehat{u} ; \cdot)$. Finally, we want to point out that strict semidifferentiability of $F$ at $u^{*}$ with respect to $W=\mathbb{R}^{n}$ is actually equivalent to strict differentiability of $F$ at $u^{*}$, see [27, Theorem 2]. For arbitrary sets $W \subset \mathbb{R}^{n}$, however, this correspondence is lost in general, see also the discussion that follows the next result.

Lemma 1 Let a cone $C \subset \mathbb{R}^{n}$, a set $W \subset \mathbb{R}^{n}$, a point $\widehat{u} \in W$, and a mapping $F: \mathbb{R}^{n} \rightarrow \mathbb{R}^{m}$ be given. Then, the following two statements hold:

a) $F$ is semidifferentiable at $\widehat{u}$ for $C$ if and only if there exists a continuous and positively homogeneous mapping $H: C \rightarrow \mathbb{R}^{m}$ such that for all $v \in C$,

$$
\left\|F\left(\widehat{u}+t v^{\prime}\right)-F(\widehat{u})-t H\left(v^{\prime}\right)\right\|=o(t) \quad \text { as } t \downarrow 0 \text { and } v^{\prime} \stackrel{C}{\rightarrow} v .
$$

Moreover, $H(\cdot)$ necessarily coincides with $F^{\prime}(\widehat{u} ; \cdot)$ given by $(6)$.

b) Suppose that $C$ is closed. Then, $F$ is strictly semidifferentiable at $\widehat{u}$ with respect to $W$ for $C$ if and only if there exists a continuous and positively homogeneous mapping $H: \mathbb{R}^{n} \rightarrow \mathbb{R}^{m}$ such that for each $v \in C$,

$$
\left\|F\left(u+t v^{\prime}\right)-F(u)-t H\left(v^{\prime}\right)\right\|=o(t) \quad \text { as } u \stackrel{W}{\rightarrow} \widehat{u}, t \downarrow 0, \text { and } v^{\prime} \rightarrow v .
$$

Moreover, $H(\cdot)$ necessarily coincides on $C$ with $F^{\prime}(\widehat{u} ; \cdot)$ given by (6).

Proof We only prove b), as a) can be proven by a similar (and even simpler) argument. Suppose that $F$ is strictly semidifferentiable at $\widehat{u}$ with respect to $W$ for $C$. Then, it follows from the discussion above that the mapping $F^{\prime}(\hat{u} ; \cdot)$ is well-defined on $C$ by (6). Clearly, this mapping is positively homogeneous. To see that $F^{\prime}(\hat{u} ; \cdot)$ is also continuous, pick $v, v^{\prime} \in C$ arbitrarily. Then, we obtain from (6) that

$$
\begin{aligned}
t\left\|F^{\prime}\left(\hat{u} ; v^{\prime}\right)-F^{\prime}(\hat{u} ; v)\right\|= & \left\|\left(F\left(\widehat{u}+t v^{\prime}\right)-F(\widehat{u})\right)-(F(\widehat{u}+t v)-F(\widehat{u}))\right\|+o(t) \\
\leq & \left\|F\left(\widehat{u}+t v^{\prime}\right)-F(\widehat{u})-t F^{\prime}(\widehat{u} ; v)\right\| \\
& +\left\|F(\widehat{u}+t v)-F(\widehat{u})-t F^{\prime}(\widehat{u} ; v)\right\|+o(t) \\
= & o(t) \quad \text { as } t \downarrow 0 \text { and } v^{\prime} \stackrel{C}{\rightarrow} v,
\end{aligned}
$$


holds true. Now, according to Tietze's extension theorem [6, Theorem 9.23], there exists a continuous mapping $\widetilde{H}: \mathbb{S} \rightarrow \mathbb{R}^{m}$ such that $\widetilde{H}(v)=F^{\prime}(\widehat{u} ; v)$ for all $v \in C \cap \mathbb{S}$. Define $H: \mathbb{R}^{n} \rightarrow \mathbb{R}^{m}$ by $H(v)=\|v\| \widetilde{H}(v /\|v\|)$ for $v \neq 0, H(0)=0$. By construction, this mapping is continuous and positively homogeneous, and by Definition 3 ,

$$
\begin{aligned}
\left\|F\left(u+t v^{\prime}\right)-F(u)-t H\left(v^{\prime}\right)\right\| & \leq\left\|F\left(u+t v^{\prime}\right)-F(u)-t F^{\prime}(\widehat{u} ; v)\right\|+t\left\|H\left(v^{\prime}\right)-H(v)\right\| \\
& =o(t) \quad \text { as } u \stackrel{W}{\rightarrow} \widehat{u}, t \downarrow 0, \text { and } v^{\prime} \rightarrow v \in C
\end{aligned}
$$

is valid.

To see that the converse implication is fulfilled, we put $F^{\prime}(\widehat{u} ; v):=H(v)$ for $v \in C$, and simply employ the stated properties of mapping $H$.

Observe that according to the previous lemma, Definition 3 reduces to Definition 2, if $C$ is closed, and $W$ is a singleton.

As a simple example, let us consider the nondifferentiable function $F: \mathbb{R} \rightarrow \mathbb{R}$ with $F(u):=|u|$. This function is semidifferentiable at 0 , and hence, strictly semidifferentiable at 0 with respect to $F^{-1}(0)$, because $F^{-1}(0)=\{0\}$ is a singleton. Function $F: \mathbb{R}^{2} \rightarrow \mathbb{R}$ with $F(u):=\left|u_{1}\right|$, is also strictly semidifferentiable at every point of $F^{-1}(0)=\{u \in$ $\mathbb{R}^{2} \mid u_{1}=0$ \} with respect to $F^{-1}(0)$, even though $F^{-1}(0)$ is not a singleton. More interesting examples related to reformulations of complementarity systems can be found in [15].

The following result is an immediate consequence of the definitions above.

Lemma 2 Let a cone $C \subset \mathbb{R}^{n}$, a mapping $F: \mathbb{R}^{n} \rightarrow \mathbb{R}^{m}$ and $\widehat{u} \in F^{-1}(0)$ be given, and consider $f$ defined according to (4). Then, the following two statements hold:

a) If $F$ is semidifferentiable at $\widehat{u}$ for $C$, so too is $f$ with $f^{\prime}(\widehat{u} ; \cdot):=\left\|F^{\prime}(\widehat{u} ; \cdot)\right\|$.

b) For any set $W \subset F^{-1}(0)$ such that $\widehat{u} \in W$, if $F$ is strictly semidifferentiable at $\widehat{u}$ with respect to $W$ for $C$, so too is $f$ with $f^{\prime}(\widehat{u} ; \cdot)$ as in a).

Our characterizations of a local $\Omega$-error bound will employ the notions of Bouligand's tangent cone and Clarke's regular tangent cone, see [34, Definition 11.1.1] for example. The tangent cone to a set $W \subset \mathbb{R}^{n}$ at $u \in W$ is given by

$$
T_{W}(u):=\left\{v \in \mathbb{R}^{n} \mid \exists\left(t_{k}\right) \downarrow 0 \exists\left(v^{k}\right) \rightarrow v:\left(u+t_{k} v^{k}\right) \subset W\right\},
$$

whereas the regular tangent cone to $W$ at $u$ is

$$
\widehat{T}_{W}(u):=\left\{v \in \mathbb{R}^{n} \mid \forall\left(u^{k}\right) \stackrel{W}{\rightarrow} u \forall\left(t_{k}\right) \downarrow 0 \exists\left(v^{k}\right) \rightarrow v:\left(u^{k}+t_{k} v^{k}\right) \subset W\right\} .
$$

It is easy to see [33, Theorem 6.26] that

$$
\widehat{T}_{W}(u) \subset T_{W}(u) .
$$

Moreover, for any $W_{1} \subset W_{2} \subset \mathbb{R}^{n}$ with $u \in W_{1}$, we have $T_{W_{1}}(u) \subset T_{W_{2}}(u)$. Note that the corresponding regular tangent cones do not satisfy such a relation in general. 
In order to approximate $T_{U}\left(u^{*}\right)$ and $\widehat{T}_{U}\left(u^{*}\right)$ later on, we consider the cones

$$
\begin{aligned}
\widehat{\mathfrak{D}}_{U} f_{G}\left(u^{*}\right) & :=\left\{v \in T_{\Omega}\left(u^{*}\right) \mid f\left(u^{k}+t_{k} v\right)=o\left(t_{k}\right) \forall\left(u^{k}\right) \stackrel{U}{\rightarrow} u^{*} \forall\left(t_{k}\right) \downarrow 0\right\}, \\
\mathfrak{D} f_{G}\left(u^{*}\right) & :=\left\{v \in T_{\Omega}\left(u^{*}\right) \mid \exists\left(t_{k}\right) \downarrow 0: f\left(u^{*}+t_{k} v\right)=o\left(t_{k}\right)\right\}, \\
\mathfrak{D} f_{H}\left(u^{*}\right) & :=\left\{v \in T_{\Omega}\left(u^{*}\right) \mid \exists\left(t_{k}\right) \downarrow 0 \exists\left(v^{k}\right) \stackrel{T_{\Omega}\left(u^{*}\right)}{\longrightarrow} v: f\left(u^{*}+t_{k} v^{k}\right)=o\left(t_{k}\right)\right\}, \\
\mathfrak{D}_{U} f_{H}\left(u^{*}\right) & :=\left\{v \in T_{\Omega}\left(u^{*}\right) \mid \begin{array}{l}
\exists\left(u^{k}\right) \stackrel{U}{\rightarrow} u^{*} \exists\left(t_{k}\right) \downarrow 0 \exists\left(v^{k}\right) \rightarrow v: \\
v^{k} \in T_{\Omega}\left(u^{k}\right) \forall k, f\left(u^{k}+t_{k} v^{k}\right)=o\left(t_{k}\right)
\end{array}\right\},
\end{aligned}
$$

where the index $G$ indicates the relation to (lower) directional Gâteaux derivatives and $H$ to (lower) directional Hadamard derivatives of $f$, cf. [34, Definition 3.1.1]. Note that those derivatives appear under different names in literature [24, 26, 27]. Some relations between these cones are established in the remaining part of this section.

Lemma 3 It holds that

$$
\widehat{\mathfrak{D}}_{U} f_{G}\left(u^{*}\right) \subset \mathfrak{D} f_{G}\left(u^{*}\right) \subset \mathfrak{D} f_{H}\left(u^{*}\right) \subset \mathfrak{D}_{U} f_{H}\left(u^{*}\right) .
$$

Proof These inclusions readily follow from the definitions of the cones involved.

Lemma 4 Suppose that, for all $v \in T_{\Omega}\left(u^{*}\right)$,

$$
\left|f\left(u^{*}+t v^{\prime}\right)-f\left(u^{*}+t v\right)\right|=o(t) \quad \text { as } t \downarrow 0 \text { and } v^{\prime} \stackrel{T_{\Omega}\left(u^{*}\right)}{\longrightarrow} v,
$$

is valid. Then, $\mathfrak{D} f_{G}\left(u^{*}\right)=\mathfrak{D} f_{H}\left(u^{*}\right)$ holds.

Proof Because of Lemma 3, only $\mathfrak{D} f_{H}\left(u^{*}\right) \subset \mathfrak{D} f_{G}\left(u^{*}\right)$ has to be shown. Therefore, take any $v \in \mathfrak{D} f_{H}\left(u^{*}\right)$. Then, sequences $\left(t_{k}\right) \downarrow 0$ and $\left(v^{k}\right) \stackrel{T_{\Omega}\left(u^{*}\right)}{\longrightarrow} v$ exist such that

$$
f\left(u^{*}+t_{k} v^{k}\right)=o\left(t_{k}\right) .
$$

Hence, (8)-(9) yield

$$
0 \leq f\left(u^{*}+t_{k} v\right) \leq\left|f\left(u^{*}+t_{k} v\right)-f\left(u^{*}+t_{k} v^{k}\right)\right|+f\left(u^{*}+t_{k} v^{k}\right)=o\left(t_{k}\right),
$$

which implies $v \in \mathfrak{D} f_{G}\left(u^{*}\right)$.

We remark that (8) particularly holds for all $v \in T_{\Omega}\left(u^{*}\right)$, if $f$ is Lipschitz-continuous on $u^{*}+\left(T_{\Omega}\left(u^{*}\right) \cap \varepsilon \mathbb{B}\right)$ for some $\varepsilon>0$, or if $f$ is semidifferentiable at $u^{*}$ for $T_{\Omega}\left(u^{*}\right)$.

Lemma 5 The following statements are valid:

a) If $f$ is semidifferentiable at $u^{*}$ for $T_{\Omega}\left(u^{*}\right)$, then

$$
\mathfrak{D} f_{G}\left(u^{*}\right)=\mathfrak{D} f_{H}\left(u^{*}\right)=\left\{v \in T_{\Omega}\left(u^{*}\right) \mid f^{\prime}\left(u^{*} ; v\right)=0\right\} .
$$

b) If $f$ is strictly semidifferentiable at $u^{*}$ with respect to $U$ for $T_{\Omega}\left(u^{*}\right)$, then

$$
\widehat{\mathfrak{D}}_{U} f_{G}\left(u^{*}\right)=\mathfrak{D}_{U} f_{H}\left(u^{*}\right)
$$

holds in addition to (10). 
Proof We only prove assertion b), since assertion a) can be shown by a similar argument. We begin with defining the cones

$$
\begin{aligned}
& C_{1}:=\left\{v \in T_{\Omega}\left(u^{*}\right) \mid f\left(u^{k}+t_{k} v^{k}\right)=o\left(t_{k}\right) \forall\left(u^{k}\right) \stackrel{U}{\rightarrow} u^{*} \forall\left(t_{k}\right) \downarrow 0 \forall\left(v^{k}\right) \rightarrow v\right\}, \\
& C_{2}:=\left\{v \in T_{\Omega}\left(u^{*}\right) \mid \exists\left(u^{k}\right) \stackrel{U}{\rightarrow} u^{*} \exists\left(t_{k}\right) \downarrow 0 \exists\left(v^{k}\right) \rightarrow v: f\left(u^{k}+t_{k} v^{k}\right)=o\left(t_{k}\right)\right\} .
\end{aligned}
$$

Then, on the one hand, taking into account Lemma 3, the following inclusions are valid without any semidifferentiability assumptions:

$$
C_{1} \subset \widehat{\mathfrak{D}}_{U} f_{G}\left(u^{*}\right) \subset \mathfrak{D}_{U} f_{H}\left(u^{*}\right) \subset C_{2} .
$$

On the other hand, the strict semidifferentiability assumption on $f$ gives

$$
C_{1}=C_{2}=\left\{v \in T_{\Omega}\left(u^{*}\right) \mid f^{\prime}\left(u^{*} ; v\right)=0\right\} .
$$

This implies that all sets in the inclusion chain (11) are identical and moreover, coincide with the set in the right-hand side of (10).

\section{Main Results}

We start this section with a simple interpretation of a local $\Omega$-error bound, to be used below. For a given nonempty set $W \subset \mathbb{R}^{n}$, we define the projector onto $W, P_{W}: \mathbb{R}^{n} \rightrightarrows W$ by

$$
P_{W}(u):=\{\bar{u} \in W \mid \operatorname{dist}[u, W]=\|u-\bar{u}\|\} .
$$

Furthermore, we define the cone

$$
\mathfrak{C}_{U} f\left(u^{*}\right):=\left\{\begin{array}{l|l}
v \in \mathbb{R}^{n} & \begin{array}{l}
\exists\left(u^{k}\right) \stackrel{\Omega}{\rightarrow} u^{*} \exists\left(t_{k}\right) \downarrow 0 \exists\left(\bar{u}^{k}\right): \\
\bar{u}^{k} \in P_{U}\left(u^{k}\right) \forall k, \frac{u^{k}-\bar{u}^{k}}{t_{k}} \rightarrow v, \frac{f\left(u^{k}\right)}{t_{k}} \rightarrow 0
\end{array}
\end{array}\right\}
$$

Observe that $\mathfrak{C}_{U} f\left(u^{*}\right)$ is nonempty, as it contains 0 at least.

Lemma 6 The equality $\mathfrak{C}_{U} f\left(u^{*}\right)=\{0\}$ holds if and only if $f$ provides a local $\Omega$-error bound at $u^{*}$ for $U$.

Proof This follows immediately from the definitions. (Note that this lemma can also be derived from [35, Theorem 3.1].)

\subsection{Necessary Conditions for an Error Bound}

In this subsection, we derive necessary conditions for the error bound, which essentially have the form of lower approximations of the (regular) tangent cone to the set $U$ at $u^{*}$. Our constructions here, as well as those in the subsequent Section 3.2, do not employ any (semi)differentiability assumptions. Moreover, we provide conditions ensuring Clarke regularity of the solution set $U$ at $u^{*}$ under the local $\Omega$-error bound. One of these conditions is the following property of the constraint set $\Omega$.

Definition 4 The set $\Omega$ is called $T$-conical near $\widehat{u} \in \Omega$ if there exists $\delta>0$ such that

$$
\Omega \cap(\widehat{u}+\delta \mathbb{B})=\widehat{u}+\left(T_{\Omega}(\widehat{u}) \cap \delta \mathbb{B}\right) .
$$


An example of sets that are $T$-conical near any of their elements are closed semilinear sets (i.e., finite unions of closed polyhedrons), see [18, Proposition 8.24]. In fact, $\Omega$ is evidently $T$-conical near $u^{*}$ if, in some neighborhood of $u^{*}, \Omega-u^{*}$ is a closed (not necessarily convex) cone.

Remark 1 In [1, Section 2], $\Omega$ is called conical near $\widehat{u} \in \Omega$ provided the radial cone to $\Omega$ at $\widehat{u}$, denoted by $R_{\Omega}(\widehat{u})$, coincides with $\Omega-\widehat{u}$ near $\widehat{u}$. It is known [34, Proposition 11.1.2 (a)] that $R_{\Omega}(\widehat{u}) \subset T_{\Omega}(\widehat{u})$. If $\Omega$ is convex, then $T_{\Omega}(\widehat{u})$ equals the closure of $R_{\Omega}(\widehat{u})$ [34, Proposition 11.1.2 (d)]. This implies that these two conicity properties are the same for closed convex sets. In general, however, it can be shown that $T$-conicity is stronger than conicity. In particular, unlike conicity, $T$-conicity implies closedness of $\Omega$ near $\widehat{u}$ since $T_{\Omega}(\widehat{u})$ is always closed [33, Proposition 6.2].

Lemma 7 If $\Omega$ is $T$-conical near $\widehat{u} \in \Omega$, i.e., (12) holds with some $\delta>0$, then the following statements are equivalent:

a) $\Omega \cap(\widehat{u}+\delta \mathbb{B})+t v \subset \widehat{u}+T_{\Omega}(\widehat{u})$ for all $v \in T_{\Omega}(\widehat{u}), t \in[0, \infty)$.

b) $\widehat{T}_{\Omega}(\widehat{u})=T_{\Omega}(\widehat{u})$.

Proof In order to prove that a) implies b), since $\Omega$ is closed near $\widehat{u}$ (see Remark 1), we can employ [32, formula (2.1)]: $v \in \widehat{T}_{\Omega}(\widehat{u})$ if and only if for every $\gamma>0$ there are $\lambda>0$ and $\mu>0$ such that

$$
\Omega \cap(u+t(v+\gamma \mathbb{B})) \neq \varnothing \quad \text { for all } u \in \Omega \cap(\widehat{u}+\lambda \mathbb{B}), t \in[0, \mu] .
$$

Let us now assume that $\widehat{v} \in T_{\Omega}(\widehat{u})$ exists with $\widehat{v} \notin \widehat{T}_{\Omega}(\widehat{u})$. Then, due to (13), there are $\gamma>0$ and sequences $\left(u^{k}\right) \stackrel{\Omega}{\rightarrow} \widehat{u},\left(t_{k}\right) \downarrow 0$ such that, for all $k \in \mathbb{N}$,

$$
\Omega \cap\left(u^{k}+t_{k}(\widehat{v}+\gamma \mathbb{B})\right)=\emptyset .
$$

Taking into account local closedness of $\Omega$ again, (14) is equivalent to

$$
\operatorname{dist}\left[u^{k}+t_{k}(\widehat{v}+\gamma \mathbb{B}), \Omega\right]>0
$$

for all $k \in \mathbb{N}$ sufficiently large, where

$$
\operatorname{dist}\left[W_{1}, W_{2}\right]:=\inf \left\{\left\|w^{1}-w^{2}\right\| \mid w^{1} \in W_{1}, w^{2} \in W_{2}\right\}
$$

for nonempty sets $W_{1}, W_{2} \subset \mathbb{R}^{n}$. Hence,

$$
\operatorname{dist}\left[u^{k}+t_{k} \widehat{v}, \Omega\right]>0
$$

follows for all $k \in \mathbb{N}$ large enough. Because $\Omega$ is $T$-conical near $\widehat{u},\left(u^{k}\right) \stackrel{\Omega}{\rightarrow} \widehat{u}$, and $\left(t_{k}\right) \downarrow 0$, the previous inequality yields

$$
\operatorname{dist}\left[u^{k}+t_{k} \widehat{v}, \widehat{u}+T_{\Omega}(\widehat{u})\right]>0
$$

for all $k \in \mathbb{N}$ large enough. This contradicts a).

Let us now demonstrate that b) implies a). Since $\Omega$ is $T$-conical near $\widehat{u}$, we obtain from b) that

$$
\Omega \cap(\widehat{u}+\delta \mathbb{B})=\widehat{u}+\left(\widehat{T}_{\Omega}(\widehat{u}) \cap \delta \mathbb{B}\right) .
$$

Therefore, by the convexity of the regular tangent cone [33, Theorem 6.26], we have

$$
\Omega \cap(\widehat{u}+\delta \mathbb{B})+t v=\widehat{u}+t v+\left(\widehat{T}_{\Omega}(\widehat{u}) \cap \delta \mathbb{B}\right) \subset \widehat{u}+\widehat{T}_{\Omega}(\widehat{u})
$$


for any $v \in \widehat{T}_{\Omega}(\widehat{u})$ and any $t \geq 0$.

Remark 2 If $\Omega$ is closed near $\widehat{u}$, property b) in Lemma 7 is known as Clarke regularity of $\Omega$ at $\widehat{u}$ [33, Corollary 6.29], and it is automatically fulfilled provided $\Omega$ is convex [33, Theorem 6.9], for instance.

\section{Lemma 8 Let}

$$
\Omega \cap\left(u^{*}+\delta \mathbb{B}\right) \subset u^{*}+T_{\Omega}\left(u^{*}\right)
$$

be satisfied for some $\delta>0$ (which always holds if $\Omega$ is $T$-conical near $u^{*}$, or if $\Omega$ is convex). Then,

$$
T_{U}\left(u^{*}\right)=\left\{v \in T_{\Omega}\left(u^{*}\right) \mid \exists\left(t_{k}\right) \downarrow 0 \exists\left(v^{k}\right) \stackrel{T_{\Omega}\left(u^{*}\right)}{\longrightarrow} v:\left(u^{*}+t_{k} v^{k}\right) \subset U\right\}
$$

and, as a consequence,

$$
T_{U}\left(u^{*}\right) \subset \mathfrak{D} f_{H}\left(u^{*}\right)
$$

are valid.

Proof Since $U \subset \Omega$, we have $T_{U}\left(u^{*}\right) \subset T_{\Omega}\left(u^{*}\right)$. Therefore,

$$
T_{U}\left(u^{*}\right)=\left\{v \in T_{\Omega}\left(u^{*}\right) \mid \exists\left(t_{k}\right) \downarrow 0 \exists\left(v^{k}\right) \rightarrow v:\left(u^{*}+t_{k} v^{k}\right) \subset U\right\}
$$

follows. Now, pick any $v \in T_{U}\left(u^{*}\right)$. Because of (18), there are sequences $\left(t_{k}\right) \downarrow 0$ and $\left(v^{k}\right) \rightarrow v$ such that $\left(u^{*}+t_{k} v^{k}\right) \subset U$, and we obtain for all $k \in \mathbb{N}$ sufficiently large that

$$
u^{*}+t_{k} v^{k} \in U \cap\left(u^{*}+\delta \mathbb{B}\right) \subset \Omega \cap\left(u^{*}+\delta \mathbb{B}\right) \subset u^{*}+T_{\Omega}\left(u^{*}\right),
$$

where the last inclusion is by (15). Thus, we have $v^{k} \in T_{\Omega}\left(u^{*}\right)$ for all $k \in \mathbb{N}$ large enough, and (16) follows. Finally, since $u^{*}+t_{k} v^{k} \in U$ implies $f\left(u^{*}+t_{k} v^{k}\right)=0$, inclusion (17) becomes obvious.

Theorem 1 Let $\Omega$ be $T$-conical near $u^{*}$, and suppose that $f$ provides a local $\Omega$-error bound at $u^{*}$ for $U$. Then, the following statements are valid:

a) $\mathfrak{D} f_{G}\left(u^{*}\right) \subset T_{U}\left(u^{*}\right)$.

b) $\widehat{T}_{\Omega}\left(u^{*}\right)=T_{\Omega}\left(u^{*}\right)$ implies $\widehat{\mathfrak{D}}_{U} f_{G}\left(u^{*}\right) \subset \widehat{T}_{U}\left(u^{*}\right)$.

c) If, in addition to the equality $\widehat{T}_{\Omega}\left(u^{*}\right)=T_{\Omega}\left(u^{*}\right)$, it holds that

$$
\widehat{\mathfrak{D}}_{U} f_{G}\left(u^{*}\right)=\mathfrak{D} f_{H}\left(u^{*}\right),
$$

then $\widehat{T}_{U}\left(u^{*}\right)=T_{U}\left(u^{*}\right)$ is valid.

Proof We first prove a). Take any $v \in \mathfrak{D} f_{G}\left(u^{*}\right)$. Then, $v \in T_{\Omega}\left(u^{*}\right)$, and there is a sequence $\left(t_{k}\right) \downarrow 0$ such that $f\left(u^{*}+t_{k} v\right)=o\left(t_{k}\right)$. Because $\Omega$ is $T$-conical near $u^{*}$, it follows that $u^{*}+t_{k} v \in \Omega$ for all $k \in \mathbb{N}$ sufficiently large. Then, since $f$ provides a local $\Omega$-error bound at $u^{*}$ for $U$, there exists $c>0$ such that

$$
c \operatorname{dist}\left[u^{*}+t_{k} v, U\right] \leq f\left(u^{*}+t_{k} v\right)=o\left(t_{k}\right)
$$

for $k \in \mathbb{N}$ large enough. Evidently, the latter implies $v \in T_{U}\left(u^{*}\right)$.

Let us now prove b). Since $U \subset \Omega$, Lemma 7 yields that, for any $v \in T_{\Omega}\left(u^{*}\right)$ and any $t \geq 0$,

$$
U \cap\left(u^{*}+\delta \mathbb{B}\right)+t v \subset u^{*}+T_{\Omega}\left(u^{*}\right)
$$


holds. Then, (20) is particularly valid for all $v \in \widehat{\mathfrak{D}}_{U} f_{G}\left(u^{*}\right) \subset T_{\Omega}\left(u^{*}\right)$. Therefore, for any fixed $\widehat{v} \in \widehat{\mathfrak{D}}_{U} f_{G}\left(u^{*}\right)$, we have

$$
u^{k}+t_{k} \widehat{v} \in u^{*}+T_{\Omega}\left(u^{*}\right)
$$

for arbitrary sequences $\left(u^{k}\right) \stackrel{U}{\rightarrow} u^{*}$ and $\left(t_{k}\right) \downarrow 0$, provided $k \in \mathbb{N}$ is large enough. Thus, similar to the proof of a), $T$-conicity of $\Omega$ and the local $\Omega$-error bound yield

$$
c \operatorname{dist}\left[u^{k}+t_{k} \widehat{v}, U\right] \leq f\left(u^{k}+t_{k} \widehat{v}\right)=o\left(t_{k}\right) .
$$

Hence, $\widehat{v} \in \widehat{T}_{U}\left(u^{*}\right)$ is valid.

It remains to prove c). Due to (7), just $T_{U}\left(u^{*}\right) \subset \widehat{T}_{U}\left(u^{*}\right)$ needs to be shown. By (17) in Lemma 8 and assumption (19), we have $T_{U}\left(u^{*}\right) \subset \mathfrak{D} f_{H}\left(u^{*}\right)=\widehat{\mathfrak{D}}_{U} f_{G}\left(u^{*}\right)$. Now, statement b) of this theorem completes the proof.

The following example shows that $T$-conicity cannot be removed in general from the assumptions in Theorem 1.

Example 1 Let $\Omega:=((1,0)+\mathbb{B}) \cap \mathbb{R}_{+}^{2}$. Consider $f(u):=u_{1}+u_{2}$ for $u \in \Omega$, and $f(u):=0$ otherwise. Then, $U=f^{-1}(0) \cap \Omega=\{0\}$. For $u^{*}=0$, it is easily seen that $\widehat{T}_{U}\left(u^{*}\right)=T_{U}\left(u^{*}\right)=\{0\}$. Moreover, we have $\widehat{T}_{\Omega}\left(u^{*}\right)=T_{\Omega}\left(u^{*}\right)=\mathbb{R}_{+}^{2}$. Hence, $\Omega$ is not $T$-conical near $u^{*}$. Now, short computations give $\widehat{\mathfrak{D}}_{U} f_{G}\left(u^{*}\right)=\mathfrak{D} f_{G}\left(u^{*}\right)=\{0\} \times \mathbb{R}_{+}$. Therefore, $\mathfrak{D} f_{G}\left(u^{*}\right) \not \subset T_{U}\left(u^{*}\right)$ and $\widehat{\mathfrak{D}}_{U} f_{G}\left(u^{*}\right) \not \subset \widehat{T}_{U}\left(u^{*}\right)$. Since $f$ coincides with the $l_{1}$ norm on $\Omega$, it provides an even global $\Omega$-error bound at $u^{*}$. Therefore, the inclusions in Theorem $1 \mathrm{a}$ ) and b) fail to hold just because $\Omega$ is not $T$-conical near $u^{*}$.

In order to show that statement c) in Theorem 1 is also not valid without $T$-conicity of $\Omega$ near $u^{*}$, we will have to slightly modify the function $f$ defined above. Take any sequence $\left(u^{k}\right) \subset((1,0)+\mathbb{S}) \cap \mathbb{R}_{+}^{2}$ convergent to $u^{*}=0$, and with $u^{k} \neq u^{*}$ for all $k \in \mathbb{N}$ (e.g., set $u^{k}=(1 / k, \sqrt{2 k-1} / k)$; see Fig. 1). Consider re-defined $f$ at the points of this sequence by setting $f\left(u^{k}\right):=0$ for all $k \in \mathbb{N}$. Then $U=f^{-1}(0) \cap \Omega=\left(u^{k}\right) \cup\{0\}$, and this set is closed, but not Clarke regular at $u^{*}=0$, as $T_{U}\left(u^{*}\right)=\{0\} \times \mathbb{R}_{+}$, while $\widehat{T}_{U}\left(u^{*}\right)=\{0\}$. At

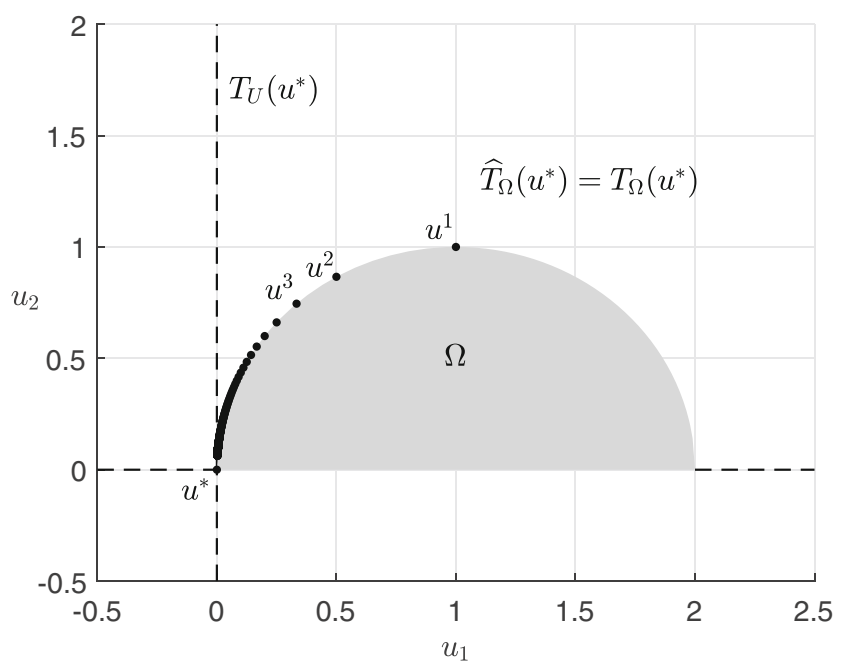

Fig. 1 Constraint set, solutions, and related cones in Example 1 
the same time, it can be easily seen that $\widehat{\mathfrak{D}}_{U} f_{G}\left(u^{*}\right)=\mathfrak{D} f_{H}\left(u^{*}\right)=\{0\} \times \mathbb{R}_{+}$. Moreover, for the same reason as above, $f$ provides an $\Omega$-error bound at $u^{*}$.

Theorem 2 Suppose that $\Omega$ is closed and convex, and that for all $v \in T_{\Omega}\left(u^{*}\right)$,

$$
\left|f\left(u+t v^{\prime}\right)-f(u+t v)\right|=o(t) \quad \text { as } u \stackrel{U}{\rightarrow} u^{*}, t \downarrow 0, \text { and } v^{\prime} \rightarrow v \text { with } u+t v^{\prime} \in \Omega,(21)
$$

holds true. If $f$ provides a local $\Omega$-error bound at $u^{*}$ for $U$, then the following statements are valid:

a) $\mathfrak{D} f_{G}\left(u^{*}\right)=T_{U}\left(u^{*}\right)$.

b) $\widehat{\mathfrak{D}}_{U} f_{G}\left(u^{*}\right) \subset \widehat{T}_{U}\left(u^{*}\right)$.

c) $\widehat{\mathfrak{D}}_{U} f_{G}\left(u^{*}\right)=\mathfrak{D} f_{G}\left(u^{*}\right)$ implies $\widehat{T}_{U}\left(u^{*}\right)=T_{U}\left(u^{*}\right)$.

Proof Let us prove a) first. Pick any $v \in \mathfrak{D} f_{G}\left(u^{*}\right)$. Then, there exists a sequence $\left(t_{k}\right) \downarrow 0$ such that

$$
f\left(u^{*}+t_{k} v\right)=o\left(t_{k}\right)
$$

is valid. According to Remark 2, we have $\widehat{T}_{\Omega}\left(u^{*}\right)=T_{\Omega}\left(u^{*}\right)$. Hence, due to $\mathfrak{D} f_{G}\left(u^{*}\right) \subset$ $T_{\Omega}\left(u^{*}\right)$, we now obtain that there exists a sequence $\left(v^{k}\right) \rightarrow v$, such that

$$
u^{*}+t_{k} v^{k} \in \Omega
$$

holds for all $k \in \mathbb{N}$. Furthermore, (23) and the local $\Omega$-error bound imply

$$
c \operatorname{dist}\left[u^{*}+t_{k} v^{k}, U\right] \leq f\left(u^{*}+t_{k} v^{k}\right),
$$

for some $c>0$ and all $k \in \mathbb{N}$ large enough. From [33, Example 9.6], we observe that

$$
\left|\operatorname{dist}\left[u^{*}+t_{k} v^{k}, U\right]-\operatorname{dist}\left[u^{*}+t_{k} v, U\right]\right| \leq t_{k}\left\|v^{k}-v\right\|=o\left(t_{k}\right)
$$

holds true for $k \in \mathbb{N}$ sufficiently large. This, combined with (21), (22) and (24) gives

$$
\begin{aligned}
c \operatorname{dist}\left[u^{*}+t_{k} v, U\right] & \leq c \operatorname{dist}\left[u^{*}+t_{k} v^{k}, U\right]+c\left|\operatorname{dist}\left[u^{*}+t_{k} v^{k}, U\right]-\operatorname{dist}\left[u^{*}+t_{k} v, U\right]\right| \\
& \leq f\left(u^{*}+t_{k} v^{k}\right)+o\left(t_{k}\right) \\
& \leq f\left(u^{*}+t_{k} v\right)+\left|f\left(u^{*}+t_{k} v^{k}\right)-f\left(u^{*}+t_{k} v\right)\right|+o\left(t_{k}\right) \\
& =o\left(t_{k}\right) .
\end{aligned}
$$

Hence, as in the proof of Theorem 1 a), $v \in T_{U}\left(u^{*}\right)$ and thus, $\mathfrak{D} f_{G}\left(u^{*}\right) \subset T_{U}\left(u^{*}\right)$ follows. Finally, we obtain from the latter that (17) in Lemma 8 in combination with Lemma 4 yields

$$
\mathfrak{D} f_{G}\left(u^{*}\right) \subset T_{U}\left(u^{*}\right) \subset \mathfrak{D} f_{H}\left(u^{*}\right)=\mathfrak{D} f_{G}\left(u^{*}\right),
$$

showing that all cones in this chain of inclusions coincide.

Let us now prove b). Because of $\widehat{T}_{\Omega}\left(u^{*}\right)=T_{\Omega}\left(u^{*}\right)$ and $U \subset \Omega$, the argument that was used to prove that b) implies a) in Lemma 7 yields, for any $v \in T_{\Omega}\left(u^{*}\right)$ and any $t \geq 0$, that

$$
U \cap\left(u^{*}+\delta \mathbb{B}\right)+t v \subset u^{*}+t v+\left(T_{\Omega}\left(u^{*}\right) \cap \delta \mathbb{B}\right) \subset u^{*}+T_{\Omega}\left(u^{*}\right) .
$$

Now, pick $\widehat{v} \in \widehat{\mathfrak{D}}_{U} f_{G}\left(u^{*}\right)$ arbitrarily. Moreover, take any sequences $\left(u^{k}\right) \stackrel{U}{\rightarrow} u^{*}$ and $\left(t_{k}\right) \downarrow$ 0 . Then, as in the proof of statement b) in Theorem 1, we want to show that

$$
\operatorname{dist}\left[u^{k}+t_{k} \widehat{v}, U\right]=o\left(t_{k}\right)
$$

is valid. Because of

$$
\widehat{\mathfrak{D}}_{U} f_{G}\left(u^{*}\right) \subset T_{\Omega}\left(u^{*}\right)=\widehat{T}_{\Omega}\left(u^{*}\right),
$$


we obtain that (25) is particularly valid for $\widehat{v}$ and all $t \geq 0$. Moreover, (27) yields the existence of a sequence $\left(v^{k}\right) \rightarrow \widehat{v}$ so that

$$
u^{k}+t_{k} v^{k} \in \Omega
$$

for all $k \in \mathbb{N}$. Thus, similar to the proof of a), one can verify that (26) holds true.

It remains to show c). Particularly, only the inclusion $T_{U}\left(u^{*}\right) \subset \widehat{T}_{U}\left(u^{*}\right)$ has to be verified. This, however, follows immediately from a), b), and the assumption that $\widehat{\mathfrak{D}}_{U} f_{G}\left(u^{*}\right)=$ $\mathfrak{D} f_{G}\left(u^{*}\right)$.

Unlike in Theorem 2, the assumptions of Theorem 1 do not include closedness and convexity of $\Omega$. Observe, however, that taking into account Remark $1, T$-conicity of $\Omega$ near $u^{*}$ and the equality $\widehat{T}_{\Omega}\left(u^{*}\right)=T_{\Omega}\left(u^{*}\right)$ (as in item b) in Theorem 1) imply the existence of $\varepsilon>0$ such that $\Omega \cap\left(u^{*}+\varepsilon \mathbb{B}\right)$ is closed and convex.

By Example 1 above it is demonstrated that, in general, assumption (21) cannot be removed from Theorem 2. Observe that according to (25), condition (21) is automatically satisfied for all $v \in T_{\Omega}\left(u^{*}\right)$ if $f$ is Lipschitz-continuous on $u^{*}+\left(T_{\Omega}\left(u^{*}\right) \cap \varepsilon \mathbb{B}\right)$ for some $\varepsilon>0$, or if $f$ is strictly semidifferentiable at $u^{*}$ with respect to $U$ for $T_{\Omega}\left(u^{*}\right)$.

We complete this subsection by noting that our blanket assumption that $U$ is locally closed was actually never used in it (as well as in Section 2).

\subsection{Sufficient Condition for an Error Bound}

From this point on, we will make use of the normal cone to $U$ at $u^{*}$ relative to $\Omega$ [35, formula (4)], defined as

$N_{U}\left(u^{*} ; \Omega\right):=\left\{v \in \mathbb{R}^{n} \mid \exists\left(u^{k}\right) \stackrel{\Omega}{\rightarrow} u^{*} \exists\left(t_{k}\right) \downarrow 0 \exists\left(\bar{u}^{k}\right): \bar{u}^{k} \in P_{U}\left(u^{k}\right) \forall k, \frac{u^{k}-\bar{u}^{k}}{t_{k}} \rightarrow v\right\}$.

Note that $N_{U}\left(u^{*} ; \Omega\right) \subset N_{U}\left(u^{*}\right)$, where $N_{U}\left(u^{*}\right):=N_{U}\left(u^{*} ; \mathbb{R}^{n}\right)$ is nothing else than Mordukhovich's (limiting) normal cone to $U$ at $u^{*}$, see [25, Definition 1.1] or [33, Definition 6.3].

From the definitions it evidently follows that

$$
\mathfrak{C}_{U} f\left(u^{*}\right) \subset N_{U}\left(u^{*} ; \Omega\right)
$$

holds.

Lemma 9 The following inclusion is valid: $N_{U}\left(u^{*} ; \Omega\right) \subset \widehat{T}_{U}\left(u^{*}\right)^{\circ}$.

Proof Simple relations yield $N_{U}\left(u^{*} ; \Omega\right) \subset N_{U}\left(u^{*} ; \mathbb{R}^{n}\right) \subset N_{U}\left(u^{*} ; \mathbb{R}^{n}\right)^{\circ \circ}$. Moreover, $N_{U}\left(u^{*} ; \mathbb{R}^{n}\right)^{\circ}=\widehat{T}_{U}\left(u^{*}\right)$ follows from [33, Theorem 6.28(b)], and hence, we conclude that $N_{U}\left(u^{*} ; \mathbb{R}^{n}\right)^{\circ \circ}=\widehat{T}_{U}\left(u^{*}\right)^{\circ}$.

Now, we are in a position to introduce a condition which may be considered as a generalization of the notion of a noncritical solution; see the discussion in Section 4 below.

Condition $1 \widehat{T}_{U}\left(u^{*}\right)=\mathfrak{D}_{U} f_{H}\left(u^{*}\right)$.

Lemma 10 Let $\Omega \cap\left(u^{*}+\delta \mathbb{B}\right) \subset u^{*}+T_{\Omega}\left(u^{*}\right)$ be satisfied for some $\delta>0$. Then, Condition 1 is fulfilled if and only if $\widehat{T}_{U}\left(u^{*}\right)=T_{U}\left(u^{*}\right)$ and $T_{U}\left(u^{*}\right)=\mathfrak{D}_{U} f_{H}\left(u^{*}\right)$. 
Proof Evidently, if $\widehat{T}_{U}\left(u^{*}\right)=T_{U}\left(u^{*}\right)$ and $T_{U}\left(u^{*}\right)=\mathfrak{D}_{U} f_{H}\left(u^{*}\right)$, then Condition 1 is valid. Now, if Condition 1 holds true, then (7), and (17) in Lemma 8, together with Lemma 3 yield

$$
\mathfrak{D}_{U} f_{H}\left(u^{*}\right)=\widehat{T}_{U}\left(u^{*}\right) \subset T_{U}\left(u^{*}\right) \subset \mathfrak{D} f_{H}\left(u^{*}\right) \subset \mathfrak{D}_{U} f_{H}\left(u^{*}\right),
$$

showing that all cones in this chain coincide.

Theorem 3 Let $\Omega$ be convex. If Condition 1 is satisfied, and if

$$
N_{U}\left(u^{*} ; \Omega\right) \subset T_{\Omega}\left(u^{*}\right),
$$

then $f$ provides a local $\Omega$-error bound at $u^{*}$ for $U$.

Proof Let us assume that $f$ does not provide a local $\Omega$-error bound at $u^{*}$ for $U$. Then, by Lemma 6, there exists $v^{*} \in \mathfrak{C}_{U} f\left(u^{*}\right)$ with $v^{*} \neq 0$, i.e.,

$$
\exists\left(u^{k}\right) \stackrel{\Omega}{\rightarrow} u^{*} \exists\left(t_{k}\right) \downarrow 0 \exists\left(\bar{u}^{k}\right): \bar{u}^{k} \in P_{U}\left(u^{k}\right) \forall k, \frac{u^{k}-\bar{u}^{k}}{t_{k}} \rightarrow v^{*}, \frac{f\left(u^{k}\right)}{t_{k}} \rightarrow 0 .
$$

Setting

$$
v^{k}:=\frac{u^{k}-\bar{u}^{k}}{t_{k}} \quad \text { for } k \in \mathbb{N}
$$

we obtain from (31) that

$$
\frac{f\left(\bar{u}^{k}+t_{k} v^{k}\right)}{t_{k}}=\frac{f\left(u^{k}\right)}{t_{k}} \rightarrow 0 .
$$

From convexity of $\Omega$, and from (32) it follows that $v^{k} \in T_{\Omega}\left(\bar{u}^{k}\right)$ for all $k \in \mathbb{N}$, while from (29) we have $v^{*} \in N_{U}\left(u^{*} ; \Omega\right)$. Therefore, properties (30)-(33) imply the inclusion $v^{*} \in \mathfrak{D}_{U} f_{H}\left(u^{*}\right)$. Thus, due to Condition 1, we have $v^{*} \in \widehat{T}_{U}\left(u^{*}\right)$. Moreover, since $v^{*} \in$ $N_{U}\left(u^{*} ; \Omega\right)$, Lemma 9 yields $v^{*} \in \widehat{T}_{U}\left(u^{*}\right)^{\circ}$. This is a contradiction because $v^{*} \neq 0$.

If $\Omega=\mathbb{R}^{n}$, then $T_{\Omega}\left(u^{*}\right)=\mathbb{R}^{n}$, and hence, assumption (30) is automatically satisfied. Beyond the unconstrained case, assumption (30) in Theorem 3 cannot be dropped, in general; see the discussion following Corollary 1 below. However, in some constrained cases, (30) holds automatically. For instance, if $u^{*}$ is isolated in $U$, then $N_{U}\left(u^{*} ; \Omega\right)=T_{\Omega}\left(u^{*}\right)$ follows immediately from the definition of these cones. Another example appears to be typical for constrained reformulations of complementarity conditions (see [9] for instance), namely the case when $\Omega=\mathbb{R}_{+}^{n}$ (or is defined by nonnegativity restrictions on some variables), and $U$ is located on the boundary of $\Omega$. Then, (30) clearly holds at any $u^{*} \in U$.

\subsection{A Criterion for an Error Bound}

In this section, employing the strict semidifferentiability assumption, we derive a condition that is necessary and sufficient for a local $\Omega$-error bound to hold. This condition is inspired by the Mordukhovich criterion for the Aubin property, cf. [25, Theorem 5.4] and [33, Theorem 9.40].

Theorem 4 Let $f$ be strictly semidifferentiable at $u^{*}$ with respect to $U$. Then, $f$ provides a local $\Omega$-error bound at $u^{*}$ for $U$ if and only if

$$
\left\{v \in \mathbb{R}^{n} \mid f^{\prime}\left(u^{*} ; v\right)=0\right\} \cap N_{U}\left(u^{*} ; \Omega\right)=\{0\} .
$$


Proof Thanks to Lemma 6, we just need to show that

$$
\mathfrak{C}_{U} f\left(u^{*}\right)=\left\{v \in \mathbb{R}^{n} \mid f^{\prime}\left(u^{*} ; v\right)=0\right\} \cap N_{U}\left(u^{*} ; \Omega\right)
$$

is satisfied. To verify this, we first assume that $v \in \mathfrak{C}_{U} f\left(u^{*}\right)$, which means that

$$
\exists\left(u^{k}\right) \stackrel{\Omega}{\rightarrow} u^{*} \exists\left(t_{k}\right) \downarrow 0 \exists\left(\bar{u}^{k}\right): \bar{u}^{k} \in P_{U}\left(u^{k}\right) \forall k, \frac{u^{k}-\bar{u}^{k}}{t_{k}} \rightarrow v, \frac{f\left(u^{k}\right)}{t_{k}} \rightarrow 0,
$$

and $v \in N_{U}\left(u^{*} ; \Omega\right)$ holds due to (29). Moreover, the strict semidifferentiability of $f$ yields

$$
f\left(u^{k}\right)=f\left(\bar{u}^{k}+t_{k} \frac{u^{k}-\bar{u}^{k}}{t_{k}}\right)=t_{k} f^{\prime}\left(u^{*} ; v\right)+o\left(t_{k}\right)
$$

and by (35) we then have $f^{\prime}\left(u^{*} ; v\right)=0$.

Let us now assume that $f^{\prime}\left(u^{*} ; v\right)=0$ and $v \in N_{U}\left(u^{*} ; \Omega\right)$. By the latter, there exist sequences $\left(u^{k}\right) \stackrel{\Omega}{\rightarrow} u^{*},\left(t_{k}\right) \downarrow 0$, and $\left(\bar{u}^{k}\right)$ with $\bar{u}^{k} \in P_{U}\left(u^{k}\right)$ for all $k \in \mathbb{N}$, and $\left(u^{k}-\bar{u}^{k}\right) / t_{k} \rightarrow v$. Then, the equality $f^{\prime}\left(u^{*} ; v\right)=0$ and the strict semidifferentiability assumption on $f$ yield $f\left(u^{k}\right) / t_{k} \rightarrow 0$. Hence, $v \in \mathfrak{C}_{U} f\left(u^{*}\right)$ follows.

None of the implications in Theorem 4 is true, in general, in the absence of strict semidifferentiability with respect to $U$, even in the unconstrained case, and even when $f$ is differentiable at $u^{*}$, see the discussion following Corollary 3 below.

\section{Some Discussions and Special Cases}

We finally discuss the relations between the main results obtained above and some other related results, for some special cases. Let $\Omega$ be closed and convex, and hence, Clarke regular at every point.

Observe first that if $f$ is strictly semidifferentiable at $u^{*}$ with respect to $U$ for $T_{\Omega}\left(u^{*}\right)$, then, from Lemmas 3 and 5 and from (17) in Lemma 8, we have

$$
\begin{aligned}
T_{U}\left(u^{*}\right) & \subset \widehat{\mathfrak{D}}_{U} f_{G}\left(u^{*}\right)=\mathfrak{D} f_{G}\left(u^{*}\right)=\mathfrak{D} f_{H}\left(u^{*}\right)=\mathfrak{D}_{U} f_{H}\left(u^{*}\right) \\
& =\left\{v \in T_{\Omega}\left(u^{*}\right) \mid f^{\prime}\left(u^{*} ; v\right)=0\right\} .
\end{aligned}
$$

Furthermore, as already mentioned above, strict semidifferentiability of $f$ at $u^{*}$ with respect to $U$ evidently implies (21) in Theorem 2 for all $v \in T_{\Omega}\left(u^{*}\right)$. Therefore, combining Theorems 2 and 3 yields the following result.

Corollary 1 Let $\Omega$ be closed and convex, and let $f$ be strictly semidifferentiable at $u^{*}$ with respect to $U$ for $T_{\Omega}\left(u^{*}\right)$. If $f$ provides a local $\Omega$-error at $u^{*}$ for $U$, then

$$
\widehat{T}_{U}\left(u^{*}\right)=\left\{v \in T_{\Omega}\left(u^{*}\right) \mid f^{\prime}\left(u^{*} ; v\right)=0\right\} .
$$

Conversely, if (30) is satisfied, and (37) holds, then $f$ provides a local $\Omega$-error bound at $u^{*}$ for $U$.

According to (7) and (36), equality (37) implies also that both its sides coincide with $T_{U}\left(u^{*}\right)$.

Assumption (30) in the statement above (and hence, in Theorem 3) cannot be dropped; this can be demonstrated by [1, Example 1.1].

We next consider the unconstrained case. Moreover, let $f$ be defined in (4) for a given continuous mapping $F: \mathbb{R}^{n} \rightarrow \mathbb{R}^{m}$, and let $u^{*}$ be a fixed solution of (5). 
Taking into account Lemma 2, we then obtain from considerations above that a combination of Theorems 2 and 3 yields the following criterion for a local error bound.

Corollary 2 Let $F$ be strictly semidifferentiable at $u^{*}$ with respect to $F^{-1}(0)$. Then,

$$
c \operatorname{dist}\left[u, F^{-1}(0)\right] \leq\|F(u)\| \quad \text { for all } u \in u^{*}+\varepsilon \mathbb{B}
$$

holds with some constants $c, \varepsilon>0$ if and only if

$$
\widehat{T}_{F^{-1}(0)}\left(u^{*}\right)=\left\{v \in \mathbb{R}^{n} \mid F^{\prime}\left(u^{*} ; v\right)=0\right\} .
$$

This result is an extension of [20, Theorem 2], where a similar assertion was obtained assuming the stronger assumption of strict differentiability of $F$ at $u^{*}$ with respect to the solution set, with (39) naturally replaced by

$$
\widehat{T}_{F^{-1}(0)}\left(u^{*}\right)=\operatorname{ker} F^{\prime}\left(u^{*}\right) .
$$

Yet again, we emphasize that under the stated assumptions, each of the equalities (39) and (40) implies that both its sides coincide with $T_{F^{-1}(0)}\left(u^{*}\right)$.

The right-hand side of (40) is the null space of the Jacobian, and hence, (40) implies that the (regular) tangent cone to the solution set at $u^{*}$ is a linear subspace. Somehow surprisingly, this turns out to be the case in Corollary 2, even when $F$ is merely strictly semidifferentiable at $u^{*}$ with respect to the solution set.

Lemma 11 Let $F$ be strictly semidifferentiable at $u^{*}$ with respect to $F^{-1}(0)$, and assume that (38) holds with some constants $c, \varepsilon>0$. Then, $T_{F^{-1}(0)}\left(u^{*}\right)$ is a linear subspace.

Proof According to Corollary 2, $T_{F^{-1}(0)}\left(u^{*}\right)=\widehat{T}_{F^{-1}(0)}\left(u^{*}\right)$ is a convex cone. Therefore, we only need to show that $-T_{F^{-1}(0)}\left(u^{*}\right) \subset T_{F^{-1}(0)}\left(u^{*}\right)$.

Let us suppose the contrary. Then, there exists $v \in T_{F^{-1}(0)}\left(u^{*}\right)$ such that $-v \notin$ $T_{F^{-1}(0)}\left(u^{*}\right)$, and according to [20, Lemma 1], there exists $\gamma>0$ such that

$$
\operatorname{dist}\left[u^{*}-t v, F^{-1}(0)\right] \geq \gamma t
$$

holds for all $t>0$ small enough. Therefore, by the error bound (38), we have

$$
\left\|F\left(u^{*}-t v\right)\right\| \geq \gamma t
$$

for all $t>0$ small enough, perhaps with a different $\gamma>0$.

Furthermore, since $v \in T_{F^{-1}(0)}\left(u^{*}\right)$, there exist sequences $\left(w^{k}\right) \rightarrow v$ and $\left(\tau_{k}\right) \downarrow 0$ such that $u^{k}:=u^{*}+\tau_{k} w^{k} \in F^{-1}(0)$ for all $k \in \mathbb{N}$. Without loss of generality, we may assume that $\|v\|=1$, and $\left\|w^{k}\right\|=1$ for all $k \in \mathbb{N}$. Passing to subsequences, if necessary, we may also ensure that $\tau_{k+1} \leq \tau_{k} / 2$ holds for all $k \in \mathbb{N}$.

For each $k \in \mathbb{N}$, set

$$
v^{k}:=-w^{k}-\frac{\tau_{k+1}}{\tau_{k}-\tau_{k+1}}\left(w^{k}-w^{k+1}\right), \quad t_{k}:=\tau_{k}-\tau_{k+1}>0 .
$$

Then, we have

$$
\begin{aligned}
u^{k}+t_{k} v^{k} & =u^{*}+\tau_{k} w^{k}-\left(\tau_{k}-\tau_{k+1}\right) w^{k}-\tau_{k+1}\left(w^{k}-w^{k+1}\right) \\
& =u^{*}+\tau_{k+1} w^{k+1} \\
& =u^{k+1} \\
& \in F^{-1}(0)
\end{aligned}
$$


Moreover,

$$
\left\|v^{k}-(-v)\right\| \leq\left\|w^{k}-v\right\|+\left\|w^{k}-w^{k+1}\right\|,
$$

and since $\left(w^{k}\right) \rightarrow v$, the right-hand side of this inequality tends to 0 . Therefore, $\left(v^{k}\right) \rightarrow-v$, and making use of the equalities $F\left(u^{k}+t_{k} v^{k}\right)=F\left(u^{k}\right)=0$, by Definition 3 we conclude that $F^{\prime}\left(u^{*} ;-v\right)=0$. It remains to observe that by (6), the latter implies $F\left(u^{*}-t v\right)=o(t)$ as $t \downarrow 0$, which contradicts (41).

Strict semidifferentiability of $F$ at $u^{*}$ with respect to the solution set cannot be replaced in this lemma by semidifferentiability at $u^{*}$. An example, where the local error bound holds but the tangent cone to the solution set is not a linear subspace can be found in [15, Example 1].

In [20, Definition 1], assuming $F$ is differentiable at $u^{*}$, the solution $u^{*}$ of (5) is called noncritical if (40) is satisfied, and critical otherwise. Therefore, according to Corollary 2 , condition (39) can be regarded as an extension of the noncriticality concept to the case when $F$ is merely semidifferentiable at $u^{*}$.

A criterion for a local error bound in the unconstrained case can also be derived from Theorem 4.

Corollary 3 Let $F$ be strictly semidifferentiable at $u^{*}$ with respect to $F^{-1}(0)$. Then, (38) holds with some constants $c, \varepsilon>0$ if and only if

$$
\left\{v \in \mathbb{R}^{n} \mid F^{\prime}\left(u^{*} ; v\right)=0\right\} \cap N_{F^{-1}(0)}\left(u^{*}\right)=\{0\} .
$$

Thus, under strict semidifferentiability with respect to the solution set, conditions (39) and (42) are both equivalent to a local error bound, and hence, to each other.

Strict semidifferentiability of $F$ at $u^{*}$ with respect to the solution set is essential for both implications in Corollary 3 (and hence, in Theorem 4), even when $F$ is differentiable at $u^{*}$; this is demonstrated by [20, Examples 3,4]. A somehow simpler example showing that, in the absence of strict semidifferentiability, the error bound (38) does not necessarily imply (42), is provided by $F: \mathbb{R}^{2} \rightarrow \mathbb{R}$ with $F(u):=\min \left\{u_{1}, u_{2}\right\}$. Indeed, this function is semidifferentiable at $u^{*}=0$, but not strictly semidifferentiable at $u^{*}$ with respect to $F^{-1}(0)=\left(\mathbb{R}_{+} \times\{0\}\right) \cup\left(\{0\} \times \mathbb{R}_{+}\right)$. One can directly verify (or conclude by [30, Corollary following Proposition 1]) that the local error bound holds at $u^{*}$. However, (42) is clearly violated.

Assuming now again that $F$ is differentiable at $u^{*}$, condition (42) takes the form

$$
\operatorname{ker} F^{\prime}\left(u^{*}\right) \cap N_{F^{-1}(0)}\left(u^{*}\right)=\{0\} \text {. }
$$

Therefore, the latter can be regarded as an alternative definition of noncriticality of $u^{*}$, because, according to Corollary 3, under strict differentiability of $F$ at $u^{*}$ with respect to the solution set, (43) is equivalent to a local error bound, and hence, to (40). This alternative definition of noncriticality appears to be new even in the smooth case, in particular, in view of the following.

A natural question arising from considerations above is whether conditions (39) and (42) are equivalent without the assumption that $F$ is strictly semidifferentiable at $u^{*}$ with respect to the solution set. (According to Corollaries 2 and 3, under the latter assumption, each of these conditions is equivalent to a local error bound (38), and hence, they are equivalent to each other.) It can be easily seen that (39) implies (42), and moreover, (37) implies (34) in the constrained case as well, but under the additional assumption (30). However, a counterexample for the converse implication is given by $F: \mathbb{R}^{2} \rightarrow \mathbb{R}$ with $F(u):=\min \left\{u_{1}-u_{2}, u_{2}\right\}$. Indeed, this function is semidifferentiable at $u^{*}=0$, but not 
strictly semidifferentiable at $u^{*}$ with respect to $F^{-1}(0)$, and one can directly verify that (42) holds, while (39) is violated.

Now, we want to figure out whether (43) implies (40) in the case when $F$ is differentiable at $u^{*}$.

Remark 3 Suppose that the following condition holds (stronger than (43)):

$$
\operatorname{ker} F^{\prime}\left(u^{*}\right) \cap \operatorname{conv} N_{F^{-1}(0)}\left(u^{*}\right)=\{0\} .
$$

According to [33, Corollary 6.5], $N_{F^{-1}(0)}\left(u^{*}\right)$ is closed, which easily implies (e.g., by means of Caratheodory theorem) that conv $N_{F^{-1}(0)}\left(u^{*}\right)$ is also closed. Furthermore, according to [33, Theorem 6.28], $\left(\operatorname{conv} N_{F^{-1}(0)}\left(u^{*}\right)\right)^{\circ}=N_{F^{-1}(0)}\left(u^{*}\right)^{\circ}=\widehat{T}_{F^{-1}(0)}\left(u^{*}\right)$. Taking into account [5, formula (2.32)], from (44) we then get

$$
\begin{aligned}
\mathbb{R}^{n} & =\left(\operatorname{ker} F^{\prime}\left(u^{*}\right) \cap \operatorname{conv} N_{F^{-1}(0)}\left(u^{*}\right)\right)^{\circ} \\
& =\operatorname{cl}\left(\operatorname{ker} F^{\prime}\left(u^{*}\right)^{\perp}+\left(\operatorname{conv} N_{F^{-1}(0)}\left(u^{*}\right)\right)^{\circ}\right) \\
& =\operatorname{cl}\left(\operatorname{ker} F^{\prime}\left(u^{*}\right)^{\perp}+\widehat{T}_{F^{-1}(0)}\left(u^{*}\right)\right) .
\end{aligned}
$$

Under differentiability of $F$ it holds that $\widehat{T}_{F^{-1}(0)}\left(u^{*}\right) \subset T_{F^{-1}(0)}\left(u^{*}\right) \subset \operatorname{ker} F^{\prime}\left(u^{*}\right)$. Suppose that (40) does not hold. Then, since $\widehat{T}_{F^{-1}(0)}\left(u^{*}\right)$ is convex, there exists a nonzero $v \in \operatorname{ker} F^{\prime}\left(u^{*}\right) \cap \widehat{T}_{F^{-1}(0)}\left(u^{*}\right)^{\circ}$ (see, e.g., [31, Corollary 11.6.1]). We claim that this $v$ cannot belong to the right-hand side of (45). Indeed, suppose the contrary: let there exist sequences $\left(v^{k}\right) \subset \operatorname{ker} F^{\prime}\left(u^{*}\right)^{\perp}$ and $\left(w^{k}\right) \subset \widehat{T}_{F^{-1}(0)}\left(u^{*}\right)$ such that $\left(v^{k}+w^{k}\right) \rightarrow v$. For all $k \in \mathbb{N}$ we then have

$$
v^{\top}\left(v^{k}+w^{k}\right)=v^{\top} w^{k} \leq 0
$$

where the left-hand side tends to $v^{\top} v=\|v\|^{2}>0$, giving a contradiction. Therefore, (40) must hold. In particular, if $\widehat{T}_{F^{-1}(0)}\left(u^{*}\right)=T_{F^{-1}(0)}\left(u^{*}\right)$ (i.e., $F^{-1}(0)$ is Clarke regular at $u^{*}$ ), then according to [33, Corollary 6.30], $N_{F^{-1}(0)}\left(u^{*}\right)$ is convex, and hence, (43) and (44) are equivalent, implying that these conditions are also equivalent to (40) in this case.

The next example (based on [21, Example 1.62]) demonstrates that (43) may hold when (44) is violated, and (43) does not imply (40), in general.

Example 2 Define the set $\mathcal{R}:=\{ \pm 1 / k \mid k=1,2, \ldots\}$ and the function $\varphi: \mathbb{R} \rightarrow \mathbb{R}$, $\varphi(x):=\operatorname{dist}[x, \mathcal{R}]$. This function is differentiable at 0 , with $\varphi^{\prime}(0)=0$. Now define $F$ : $\mathbb{R}^{2} \rightarrow \mathbb{R}$ as $F(u):=u_{2}-\varphi\left(u_{1}\right)$. This function is everywhere continuous and differentiable at $u^{*}=0$, with $F^{\prime}\left(u^{*}\right)=(0,1)$, and in particular, $\operatorname{ker} F^{\prime}\left(u^{*}\right)=\mathbb{R} \times\{0\}$. Furthermore, $F^{-1}(0)$ coincides with the graph of $\varphi$ (the solid line in Figure 2), and thus, it can be seen that $\widehat{T}_{F^{-1}(0)}\left(u^{*}\right)=\{0\}, T_{F^{-1}(0)}\left(u^{*}\right)=\mathbb{R} \times\{0\}\left(=\operatorname{ker} F^{\prime}\left(u^{*}\right)\right)$, and $N_{F^{-1}(0)}\left(u^{*}\right)=\{v \in$ $\left.\mathbb{R}^{2}|| v_{1}|\leq| v_{2} \mid\right\}$ (see Fig. 2). In particular, (43) holds, while (40) and (44) are violated.

Getting back to the constrained setting, we complete this subsection by a comparison of the conditions discussed above with the classical sufficient condition for a local $\Omega$-error bound, namely, Robinson's regularity condition. Assuming that $F$ is differentiable at a solution $u^{*}$ of the constrained equation

$$
F(u)=0 \quad \text { s.t. } u \in \Omega,
$$

the latter has the form

$$
0 \in \text { int } F^{\prime}\left(u^{*}\right)\left(\Omega-u^{*}\right) .
$$




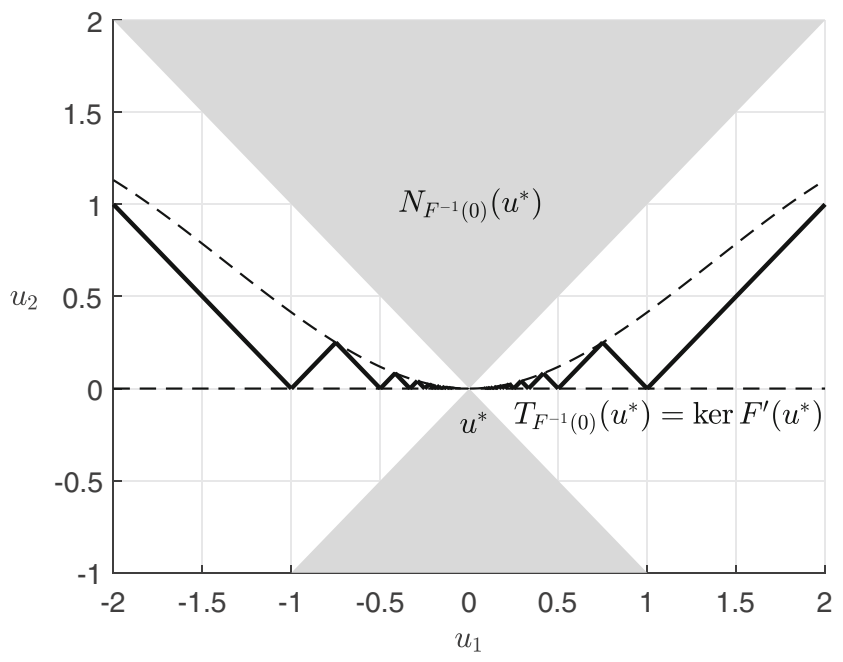

Fig. 2 Solution set and related cones in Example 2

From [29, Theorem 1], it follows that if $F$ is differentiable near $u^{*}$, with its derivative continuous at $u^{*}$, then (47) implies that $f$ defined in (4) provides a local $\Omega$-error bound at $u^{*}$. Therefore, under these smoothness assumptions, (47) certainly implies both (34) and (37) which are necessary conditions for a local $\Omega$-error bound. Observe, however, that at the same time, under any smoothness assumptions, (47) does not imply (30). This is demonstrated by the example, where $F: \mathbb{R}^{2} \rightarrow \mathbb{R}$ with $F(u):=u_{1}-u_{2}, \Omega:=\mathbb{R}_{+}^{2}$, and $u^{*}:=0$.

Furthermore, under any smoothness assumptions, and even in the unconstrained case, conditions (30), (34), and (37), do not imply (47). In order to see this, it is enough to consider $F(\cdot) \equiv 0$.

Finally, if $F$ is only assumed differentiable at $u^{*}$, (47) implies neither (34) nor (37), even in the unconstrained case when (34) reduces to (43), (37) reduces to (40), and (47) reduces to

$$
\operatorname{rank} F^{\prime}\left(u^{*}\right)=m \text {. }
$$

This is demonstrated by [20, Example 4].

\section{Application to Piecewise Strictly Semidifferentiable Equations}

In $[13,14]$, it was shown that local $\Omega$-error bounds are an important tool for achieving superlinear convergence of certain Newton-type methods applied to constrained equations of the form (46) with possibly nonisolated solutions, where $F: \mathbb{R}^{n} \rightarrow \mathbb{R}^{m}$ was assumed to be piecewise continuously differentiable. In particular, piecewise error bounds, i.e., error bounds for pieces that are active at some solution, were of interest. As before, with $f$ defined in (4), problem (46) can be written as (2), and we keep considering the solution set $U$ of the latter, and an arbitrary but fixed $u^{*} \in U$.

Let continuous functions $F^{1}, \ldots, F^{N}: \mathbb{R}^{n} \rightarrow \mathbb{R}^{m}$ be given such that $F: \mathbb{R}^{n} \rightarrow \mathbb{R}^{m}$ is continuous and satisfies

$$
F(u) \in\left\{F^{1}(u), \ldots, F^{N}(u)\right\}
$$


for every $u \in \mathbb{R}^{n}$. Then, $F^{1}, \ldots, F^{N}$ are called selection functions for $F$. Furthermore,

$$
\mathcal{A}\left(u^{*}\right):=\left\{j \mid F\left(u^{*}\right)=F^{j}\left(u^{*}\right)\right\}
$$

stands for the set of indices of all selection functions, which are active at $u^{*}$. For every $j \in \mathcal{A}\left(u^{*}\right)$, let

$$
U^{j}:=\left\{u \in \Omega \mid F^{j}(u)=0\right\}
$$

denote the solution set associated to the active selection function $F^{j}$, and define $f^{j}: \mathbb{R}^{n} \rightarrow$ $[0, \infty)$ by

$$
f^{j}(u):=\left\|F^{j}(u)\right\|
$$

Corollary 4 Suppose that $\Omega$ is closed and convex and that for all $j \in \mathcal{A}\left(u^{*}\right)$, the mapping $F^{j}$ is strictly semidifferentiable at $u^{*}$ with respect to $U^{j}$ for $T_{\Omega}\left(u^{*}\right)$. Moreover, assume that

$$
\bigcup_{j \in \mathcal{A}\left(u^{*}\right)} U^{j} \cap\left(u^{*}+\delta \mathbb{B}\right) \subset U
$$

holds true. Then, the following statements are valid:

a) If for all $j \in \mathcal{A}\left(u^{*}\right)$, the conditions

$$
\widehat{T}_{U^{j}}\left(u^{*}\right)=\left\{v \in T_{\Omega}\left(u^{*}\right) \mid\left(F^{j}\right)^{\prime}\left(u^{*} ; v\right)=0\right\}
$$

and

$$
N_{U^{j}}\left(u^{*} ; \Omega\right) \subset T_{\Omega}\left(u^{*}\right)
$$

are satisfied, then $f$ provides a local $\Omega$-error bound at $u^{*}$ for $U$.

b) Suppose that for all $j \in \mathcal{A}\left(u^{*}\right)$, the function $f^{j}$ provides a local $\Omega$-error bound at $u^{*}$ for $U^{j}$. If $F$ is strictly semidifferentiable at $u^{*}$ with respect to $U$ for $T_{\Omega}\left(u^{*}\right)$, then (37) is valid.

Proof Let us prove a) first. Due to Corollary 1 , for all $j \in \mathcal{A}\left(u^{*}\right)$ we obtain that $f^{j}$ provides a local $\Omega$-error bound at $u^{*}$ for $U^{j}$. Therefore, the assertion readily follows from [14, Propositions 2, 3].

To prove b), we notice that the mentioned propositions ensure that $f$ provides a local $\Omega$-error bound at $u^{*}$ for $U$. Thus, the assertion follows from Corollary 1 .

Inclusion (48) was crucial for the analysis in [14], as it ensures that the active solution pieces $U^{j}$ remain locally within the solution set $U$.

Clearly, one can derive a sufficient condition for $f$ to provide a local $\Omega$-error bound at $u^{*}$ for $U$, based on Theorem 4 and (48), similar to statement a) in the previous corollary. For brevity, however, we renounce to give such a result explicitly.

Funding Open Access funding enabled and organized by Projekt DEAL.

Open Access This article is licensed under a Creative Commons Attribution 4.0 International License, which permits use, sharing, adaptation, distribution and reproduction in any medium or format, as long as you give appropriate credit to the original author(s) and the source, provide a link to the Creative Commons licence, and indicate if changes were made. The images or other third party material in this article are included in the article's Creative Commons licence, unless indicated otherwise in a credit line to the material. If material is not included in the article's Creative Commons licence and your intended use is not permitted by statutory regulation or exceeds the permitted use, you will need to obtain permission directly from the copyright holder. To view a copy of this licence, visit http://creativecommonshorg/licenses/by/4.0/. 


\section{References}

1. Arutyunov, A.V., Izmailov, A.F.: Stability of possibly nonisolated solutions of constrained equations with applications to complementarity and equilibrium problems. Set-Valued Var. Anal. 26, 327-352 (2018)

2. Behling, R., Fischer, A.: A unified local convergence analysis of inexact constrained LevenbergMarquardt methods. Optim. Lett. 6, 927-940 (2012)

3. Behling, R., Fischer, A., Herrich, M., Iusem, A., Ye, Y.: A Levenberg-Marquardt method with approximate projections. Comput. Optim. Appl. 59, 5-26 (2014)

4. Behling, R., Fischer, A., Schönefeld, K., Strasdat, N.: A special complementarity function revisited. Optimization 68, 65-79 (2019)

5. Bonnans, J.F., Shapiro, A.: Perturbation Analysis of Optimization Problems. Springer, New York (2000)

6. Bruckner, A.M., Bruckner, J.B., Thomson, B.S.: Real Analysis. Prentice-Hall, Upper Saddle River (1997)

7. Burke, J.V., Ferris, M.C.: Weak sharp minima in mathematical programming. SIAM J. Control Optim. 31, 1340-1359 (1993)

8. Facchinei, F., Fischer, A., Herrich, M.: A family of Newton methods for nonsmooth constrained systems with nonisolated solution. Math. Methods Oper. Res. 77, 433-443 (2013)

9. Facchinei, F., Fischer, A., Herrich, M.: An LP-Newton method: Nonsmooth equations, KKT systems, and nonisolated solutions. Math. Program. 146, 1-36 (2014)

10. Fischer, A.: Modified Wilson's method for nonlinear programs with nonunique multipliers. Math. Oper. Res. 24, 699-727 (1999)

11. Fischer, A.: Local behavior of an iterative framework for generalized equations with nonisolated solutions. Math. Program. 94, 91-124 (2002)

12. Fischer, A.: Comments on: Critical Lagrange multipliers: What we currently know about them, how they spoil our lives, and what we can do about it. TOP 23, 27-31 (2015)

13. Fischer, A., Herrich, M.: Newton-type methods for Fritz John systems of generalized nash equilibrium problems. Pure Appl. Funct. Anal. 3, 587-602 (2018)

14. Fischer, A., Herrich, M., Izmailov, A.F., Solodov, M.V.: Convergence conditions for Newton-type methods applied to complementarity systems with nonisolated solutions. Comput. Optim. Appl. 63, 425-459 (2016)

15. Fischer, A., Jelitte, M.: On noncritical solutions of complementarity systems. In: Singh, V.K. et al. (eds.) Recent Trends in Mathematical Modeling and High Performance Computing. Springer Nature Switzerland, Cham (to appear)

16. Fischer, A., Shukla, P.K.: A Levenberg-Marquardt algorithm for unconstrained multicriteria optimization. Oper. Res. Lett. 36, 643-646 (2008)

17. Hager, W.W.: Stabilized sequential quadratic programming. Comput. Optim. Appl. 12, 253-273 (1999)

18. Ioffe, A.D.: Variational Analysis of Regular Mappings, Theory and Applications. Springer, Cham (2017)

19. Izmailov, A.F.: On the analytical and numerical stability of critical Lagrange multipliers. Comput. Math. Math. Phys. 45, 930-946 (2005)

20. Izmailov, A.F., Kurennoy, A.S., Solodov, M.V.: Critical solutions of nonlinear equations: Stability issues. Math. Program. 168, 475-507 (2018)

21. Izmailov, A.F., Solodov, M.V.: Newton-Type Methods for Optimization and Variational Problems. Springer International Publishing, Cham (2014)

22. Izmailov, A.F., Solodov, M.V.: Critical Lagrange multipliers: what we currently know about them, how they spoil our lives, and what we can do about it. TOP 23, 1-26 (2015)

23. Kanzow, C., Yamashita, N., Fukushima, M.: Levenberg-Marquardt methods with strong local convergence properties for solving nonlinear equations with convex constraints. J. Comput. Appl. Math. 172, 375-397 (2004)

24. King, A.J., Rockafellar, R.T.: Sensitivity analysis for nonsmooth generalized equations. Math. Program. 55, 193-212 (1992)

25. Mordukhovich, B.S.: Sensitivity analysis in nonsmooth optimization. In: Field, D.A., Komkov, V. (eds.) Theoretical Aspets of Industrial Design, pp. 32-46. SIAM, Philadelphia (1992)

26. Mordukhovich, B.S., Sarabi, M.E.: Critical multipliers in variational systems via second-order generalized differentiation. Math. Program. 169, 605-648 (2018)

27. Pang, J.S.: Newton's method for B-differentiable equations. Math. Oper. Res. 15, 311-341 (1990)

28. Pang, J.S.: Error bounds in mathematical programming. Math. Program. 79, 299-332 (1997)

29. Robinson, S.M.: Stability theory for systems of inequalities, Part II: Differentiable nonlinear systems. SIAM J. Numer. Anal. 13, 487-513 (1976) 
30. Robinson, S.M.: Some continuity properties of polyhedral multifunctions. Math. Program. Study 14, 206-214 (1981)

31. Rockafellar, R.T.: Convex Analysis. Princeton University Press, Princeton (1970)

32. Rockafellar, R.T.: Clarke's tangent cones and the boundaries of closed sets in $\mathbb{R}^{n}$. Nonlinear Anal. 3, 145-154 (1979)

33. Rockafellar, R.T., Wets, R.J.B.: Variational Analysis. Springer, Berlin (1998)

34. Schirotzek, W.: Nonsmooth Analysis. Springer, Heidelberg (2007)

35. Studniarski, M.: New characterizations of weak sharp and strict local minimizers in nonlinear programming. Commun. Optim. Theory 1, 19-34 (2012)

36. Wright, S.J.: Superlinear convergence of a stabilized SQP method to a degenerate solution. Comput. Optim. Appl. 11, 253-275 (1998)

37. Yamashita, N., Fukushima, M.: On the rate of convergence of the Levenberg-Marquardt method. In: Alefeld, G., Chen, X. (eds.) Topics in Numerical Analysis, Computing Supplementa, vol. 15, pp. 239249. Springer, Vienna (2001)

Publisher's Note Springer Nature remains neutral with regard to jurisdictional claims in published maps and institutional affiliations. 